\title{
A comparison between tau and amyloid- $\beta$ cerebrospinal fluid biomarkers in chronic traumatic encephalopathy and Alzheimer disease
}

Katherine W. Turk ${ }^{1,2,3 \dagger}$, Alexandra Geada ${ }^{4 \dagger}$, Victor E. Alvarez ${ }^{1,2,3,5}$, Weiming Xia ${ }^{1,5}$, Jonathan D. Cherry ${ }^{1,2,3}$, Raymond Nicks ${ }^{1,5}$, Gaoyuan Meng ${ }^{2,5}$, Sarah Daley ${ }^{1,5}$, Yorghos Tripodis ${ }^{6}$, Bertrand R. Huber ${ }^{1,2,3}$, Andrew E. Budson 1,2,3, Brigid Dwyer ${ }^{3,7}$, Neil W. Kowall 1,2,3, Robert C. Cantu 1,8,9,10,11, Lee E. Goldstein 1,12,13,14, Douglas I. Katz 3,7 , Robert A. Stern 1,3,8,10, Michael L. Alosco ${ }^{1,3}$, Jesse Mez ${ }^{1,3}$, Ann C. McKee 1,23,5,14 and Thor D. Stein ${ }^{1,2,5,14^{*}}$ (1)

\begin{abstract}
Background: Cerebrospinal fluid (CSF) tau and beta-amyloid levels in chronic traumatic encephalopathy (CTE), a disease which can be clinically indistinguishable from Alzheimer's disease (AD), are largely unknown. We examined postmortem CSF analytes among participants with autopsy confirmed CTE and AD.
\end{abstract}

Methods: In this cross-sectional study 192 participants from the Boston University AD Research Center, VA-BU-CLF Center, and Framingham Heart Study (FHS) had post-mortem CSF collected at autopsy. Participants were divided into pathological groups based on AD and CTE criteria, with 61 CTE participants (18 low, 43 high stage), 79 AD participants (23 low, 56 intermediate to high), 11 participants with CTE combined with AD, and 41 participants lacking both CTE and $A D$ neuropathology. The Meso Scale Discovery immunoassay system was utilized to measure amyloid-beta ( $A \beta_{1 .}$ $\left.{ }_{40}, A \beta_{1-42}\right)$, total tau (t-tau), and phosphorylated tau $\left(p-\operatorname{tau}_{181}\right.$ and $\left.p-\operatorname{tau}_{231}\right)$. CSF analytes were then compared across the pathological groups: no CTE/no AD (control), Low CTE, Low AD, High CTE, Intermediate/High AD, and AD+CTE.

Results: Among the Low disease state groups, the Low CTE group had significantly higher levels of $p$-tau t $_{23}$ versus the control group and compared to the Low AD group. The Low CTE group was also found to have significantly lower levels of $A \beta_{1-42}$ compared to the control group. The high CTE group had higher levels of $p$-tau ${ }_{231}$ and lower levels of $A \beta_{1-42}$ compared to Intermediate/High AD group.

Conclusions: Importantly, $\mathrm{p}$-tau $\mathrm{T}_{231}$ and $\mathrm{A} \beta_{1-42}$ were predictors of diagnosis of CTE vs. control and CTE vs. AD. Increased CSF $\mathrm{p}$-tau $\mathrm{t}_{231}$ is a promising potentially sensitive biomarker of CTE, and CSF A $\beta_{1-42}$ needs further investigation in CTE.

Keywords: Cerebrospinal fluid, Biomarkers, Chronic traumatic encephalopathy, Alzheimer's disease, Amyloid beta, Tau

\footnotetext{
*Correspondence: tdstein@bu.edu

${ }^{\dagger}$ Katherine W. Turk and Alexandra Geada contributed equally to this work.

${ }^{2}$ VA Boston Healthcare System, 150 S. Huntington Avenue, Boston, MA 02130, USA

Full list of author information is available at the end of the article
}

\section{Background}

Chronic traumatic encephalopathy (CTE) and Alzheimer disease $(\mathrm{AD})$ are neurodegenerative conditions causing memory loss that can only be definitively original author(s) and the source, provide a link to the Creative Commons licence, and indicate if changes were made. The images or other third party material in this article are included in the article's Creative Commons licence, unless indicated otherwise in a credit line to the material. If material is not included in the article's Creative Commons licence and your intended use is not permitted by statutory regulation or exceeds the permitted use, you will need to obtain permission directly from the copyright holder. To view a copy of this licence, visit http://creativecommons.org/licenses/by/4.0/. The Creative Commons Public Domain Dedication waiver (http://creativeco mmons.org/publicdomain/zero/1.0/) applies to the data made available in this article, unless otherwise stated in a credit line to the data. 
diagnosed by pathologic examination. CTE is neuropathologically distinct from $\mathrm{AD}$ and characterized by hyperphosphorylated tau ( $\mathrm{p}$-tau) deposition in neurons, astrocytes, and cell processes around the small blood vessels and at the depths of cortical sulci that often involves the superficial cortical layers II and III [1-3]. AD neuropathology consists of amyloid- $\beta$ plaques and tau neurofibrillary tangles in a distinct pattern beginning in the brainstem and entorhinal cortex in the early stages and progressing to the medial temporal lobe and finally the neocortex [4]. Analysis of differential profiles of tau-epitope phosphorylation profiles between CTE and $\mathrm{AD}$ is not yet fully understood though elevated levels of $\mathrm{p}-\mathrm{tau}_{181}$ and $\mathrm{p}-\mathrm{tau}_{231}$ have been reported in both AD and CTE $[5,6]$. Total tau (t-tau), phosphorylated tau ( $\mathrm{p}$-tau), and beta-amyloid (A $\beta)$ measurements in cerebrospinal fluid (CSF) have proven to be reliable biomarkers of $\mathrm{AD}$ and reflect changes in brain pathology that precede cognitive decline ante-mortem [7-10]. Biomarkers with the ability to detect and distinguish CTE from AD would be of great value if they show a high degree of correlation with CTE neuropathology.

Given the relatively superficial location of $\mathrm{p}$-tau in the cortex of patients with CTE compared to AD and the lack of notable involvement of amyloid $\beta$ in CTE, we hypothesized that CTE participants may have increased t-tau, p-tau ${ }_{181}$, and p-tau ${ }_{231}$ compared to cases without either CTE or AD (control), as well as relatively higher $\mathrm{p}-\operatorname{tau}_{181}, \mathrm{p}-\mathrm{tau}_{231}$, and $\mathrm{A} \beta_{42}$ concentrations compared to AD cases. Prior studies of $A \beta$ deposition in CTE [11] led us to hypothesize that CSF $A \beta_{1-40}$ would be decreased in cases with co-occurring CTE and $\mathrm{AD}$ compared to non-AD/non-CTE cases, representing a potential interaction between $\mathrm{AD}$ and $\mathrm{CTE}$. To test these hypotheses, we measured CSF levels of $A \beta_{1-40}$, $\mathrm{A} \beta_{42}$, t-tau, $\mathrm{p}$-tau ${ }_{181}$, and $\mathrm{p}$-tau ${ }_{231}$ among autopsy-confirmed participants including 61 with CTE, 79 with AD, 11 with concurrent $\mathrm{CTE}$ and $\mathrm{AD}$, and 41 control participants lacking both CTE and AD pathology. Low and high stage CTE involve different brain regions and are associated with different clinical symptoms such that high stage disease shows significant medial temporal lobe pathology and is associated with increased frequency of cognitive impairment and dementia [12]. In contrast, intermediate to high degrees of AD pathology are characterized by greater pathological involvement of the neocortex and are also associated with cognitive impairment and dementia [13]. Therefore, because the underlying neuropathology and clinical syndrome is markedly different in low and high stage disease [12], we compared CTE to AD CSF analytes separately in each disease stage.

\section{Methods}

\section{Participants}

One hundred ninety-two participants with post-mortem CSF available were enrolled from three study groups. 100 donated their brains to the Veteran's Affairs-Boston University-Concussion Legacy Foundation Brain Bank (VA-BU-CLF) as part of the Understanding Neurologic Injury and Traumatic Encephalopathy (UNITE) study, 38 were donated to the Framingham Heart Study (FHS), and 54 were donated to Boston University's Alzheimer's Disease Research Center (ADRC) as part of the Health Outreach Program for the Elderly study. The UNITE group consisted of participants with a history of exposure to contact sports such as football, ice hockey, boxing, soccer, rugby, and martial arts at either the professional or amateur level [14]. For most brain donations, the next of kin contacted the brain bank to donate tissue at or near the time of death. The participants from Boston University's Alzheimer's Disease Research Center (BU ADRC) with and without cognitive impairment underwent annual cognitive evaluations using the National Alzheimer's Disease Coordinating Center (NACC) Uniform Data Set (UDS) protocol [1]. The third cohort consisted of participants from the Framingham Heart Study (FHS), a longitudinal, community-based study. Consents for brain donation and research participation were provided by donor next of kin.

\section{Head injury exposure assessment}

For UNITE study participants, retrospective clinical evaluations were performed using semi-structured postmortem interviews, through online surveys, and review of medical records as described previously [14]. Information was obtained regarding repetitive head impact (RHI) exposure, traumatic brain injury (TBI) exposure, military history, athletic history, and clinical symptoms prior to death. In addition, medical records were examined to provide a determination of clinical symptoms and course. For the FHS participants, an athletic history assessment identical to UNITE was performed with the donor's next of kin [15]. Athletic history was not available for BU ADRC participants. All interviews were conducted independently and blinded to the results of neuropathological examination.

\section{Pathological criteria}

All brains were neuropathologically evaluated for changes consistent with CTE, AD, and other neurodegenerative disorders using previously described selection criteria and protocols. Specifically, participants were separated into pathologic groups: CTE, $\mathrm{AD}$, both (CTE+AD), or 
neither no CTE/no AD (control). Pathologic diagnosis of CTE was based on consensus criteria [1] and CTE staging I-IV was determined using previously published staging criteria $[12,16]$. Participants were stratified using NIA-Reagan criteria to high, intermediate, or low probability of dementia caused by AD, based on Braak Score and CERAD score.

There is growing recognition that with increasing age co-occurring neurodegenerative pathologies become more common $[17,18]$; therefore, comorbid neurodegenerative pathologies were not excluded from any of the groups, including the control group. Other neurodegenerative diseases were diagnosed using well-established criteria for Lewy body disease (LBD) [19] and frontotemporal lobar degeneration (FTLD) [20, 21] (Table 1). Participants with Amyotrophic Lateral Sclerosis/motor neuron disease (ALS/MND) pathology were also not excluded and included two participants in the no CTE/no AD (control) group and one participant in the high CTE group. The inclusion of a wider group of participants with concurrent neurodegenerative pathologies increases the generalizability of the current findings as patients presenting for clinical evaluation of cognitive complaints often have multiplane neurodegenerative diagnoses and underlying pathologies.

Participants were divided into pathological groups based on NIA-Reagan Criteria and CTE stage as follows. Participants with no evidence of CTE and no elements of NIA-Reagan were labeled as "No CTE/no AD" and were used and referenced as the control group throughout. Participants with CTE Stage of I-II were determined to have early stage disease and termed "Low CTE," while those with CTE Stage of III-IV were determined to have late stage disease and were termed "High CTE." Those with no evidence of CTE and NIA-Reagan of high or intermediate probability were termed "Intermediate/ High AD," while those with no evidence of CTE and NIAReagan of low probability were classified as "Low AD." Subjects with CTE and intermediate or high probability of $\mathrm{AD}$ were combined to "CTE+AD."

\section{CSF sampling and analysis}

CSF was obtained post-mortem from the foramen magnum by gently lifting the frontal lobes to access with a large bore needle. CSF was then mixed by gently inverting the tube 5 times. The tubes were centrifuged at 1500 $\mathrm{g}$ for $15 \mathrm{~min}$ at $4^{\circ} \mathrm{C}$. The CSF supernatant was removed with a transfer pipet and aliquoted into $1.5 \mathrm{~mL}$ microcentrifuge polypropylene tubes. CSF was stored at $-80 \mathrm{C}$ prior to use. CSF was then diluted 1:2 with $1 \%$ Blocker A (MSD, Rockville, MD, USA, \#R93BA) in wash buffer. Immunoassay was performed for $A \beta_{1-42}$ and $A \beta_{1-40}$, using a multiplex plate from MSD (\#K15200E), as well as for levels of p-tau ${ }_{231}$ and total tau (MSD \#K15121D) according to manufacturer's protocol. To capture tau phosphorylated at Thr residue 181, antibody AT270 was used and the detecting antibody was the biotinylated HT7 that recognizes residue 159-163 of tau (Thermo Scientific, Rockford, IL). For hemoglobin quantification, CSF was diluted 1:3000 and applied to the RayBio Human Hemoglobin ELISA kit (\# ELH-Hgb). All standards and samples were run in duplicate.

\section{Tissue sampling and analysis}

The buffer conditions, protease inhibitors, and centrifugation protocols have been reported previously [11]. A 4-mm-tissue punch was used to isolate and remove gray matter from the gyral crests and sulcal depths of the middle frontal gyrus and neighboring sulci and superior temporal gyrus and sulcus. The brain tissue was homogenized in five-fold volume of $5 \mathrm{M}$ Guanidine Hydrocholride/50 $\mathrm{mM}$ Tris-HCL, $\mathrm{pH}$ 8.0, with protease inhibitors (Thermo Scientific, 78439) and phosphatase inhibitors (Sigma, P5726 and P0044). The tissue was homogenized using a mechanical homogenizer for 25 strokes followed by ultrasonic disruption on ice. The homogenates were shaken at room temperature overnight. The lysate was diluted 1:80 with 1\% Blocker A (MSD, \#R93BA) in wash buffer, and immunoassay was performed for $A \beta_{1-42}$ using a multiplex plate from MSD.

\section{Statistical analysis}

Statistical analysis was performed using SPSS 26.0 (IBM Corp, Armonk, NY) and Prism v8 (Graph-Pad Software, La Jolla, CA). A one-way analysis of variance (ANOVA) was used to compare age among groups. Levels of $A \beta_{1-40}$, $\mathrm{A} \beta_{1-42}, \mathrm{p}-\mathrm{tau}_{181}, \mathrm{p}-\mathrm{tau}_{231}$, and total tau that were outside $3 \mathrm{X}$ the interquartile range were eliminated as outliers and included $n=1$ from no CTE/no AD (control) group, $n=1$ from low CTE group, $n=1$ high CTE group, $n=1$ from low AD group, and $n=1$ from intermediate/high AD group for ptau 181 analysis, $n=3$ from the control group, $n=1$ from low AD group, $n=1$ for the high CTE group, $n=6$ from intermediate/high AD group, and $n=1$ from CTE +AD group for ptau 231 analysis, $n=1$ from no $\mathrm{CTE} /$ no $\mathrm{AD}$ (control) group, $n=2$ from high CTE group for total tau analysis, $n=5$ from control group, $n=3$ from low CTE group, $n=4$ from high CTE group, $n=1$ from intermediate/high AD group, $n=1$ for the CTE $+\mathrm{AD}$ group for $A \beta_{1-42}$ analysis, $n=2$ from the control group, $n=1$ from low CTE group, $n=3$ from high CTE group, $n=1$ from low AD group, and $n=2$ from intermediate/ high $A D$ group for $A \beta_{1-40}$ analysis. A two-sample chisquare test weighted by sample size was used to compare the frequency of men in each pathologic group, as well as the frequency of FTLD and LBD pathologies between 
Table 1 Demographic and exposure characteristics of participant groups

\begin{tabular}{|c|c|c|c|c|c|c|c|}
\hline & No CTE/No AD & Low CTE & High CTE & Low AD & Int/High AD & $\mathrm{CTE}+\mathrm{AD}$ & $p$ \\
\hline Sample size $(n)$ & 41 & 18 & 43 & 23 & 56 & 11 & \\
\hline Age at death (S.E.M.) & $74.1(3.0)^{d}$ & $64.6(4.6)^{f, d, e}$ & $74.8(1.3)^{d}$ & $86.7(1.1)^{\mathrm{a}, \mathrm{b}, \mathrm{c}}$ & $81.1(1.3)^{b}$ & $79.6(2.8)^{\mathrm{b}}$ & $<.001$ \\
\hline Age range (max-min) & $101-17$ & $89-25$ & $90-53$ & $95-72$ & $98-54$ & $70-100$ & \\
\hline \multicolumn{8}{|l|}{ Cohort } \\
\hline FHS & 13 & 1 & 0 & 10 & 13 & 1 & \\
\hline UNITE & 17 & 17 & 43 & 1 & 12 & 10 & \\
\hline HOPE & 11 & 0 & 0 & 12 & 31 & 0 & \\
\hline Sex m/f (\%male) & $33 / 8(80.5 \%)^{c, e}$ & $18 / 0(100 \%)^{e}$ & $43 / 0(100 \%)^{\mathrm{a}, \mathrm{d}, \mathrm{e}}$ & $14 / 9(60.9 \%)^{c}$ & $27 / 29(48.2 \%)^{a, b, c}$ & 10/1 (90.9\%) & $<.001$ \\
\hline \multicolumn{8}{|l|}{ CTE stage } \\
\hline Stage I & 0 & $6(33.3 \%)$ & 0 & 0 & 0 & $1(9.1 \%)$ & \\
\hline Stage II & 0 & $12(66.7 \%)$ & 0 & 0 & 0 & $1(9.1 \%)$ & \\
\hline Stage III & 0 & 0 & $18(41.9 \%)$ & 0 & 0 & 0 & \\
\hline Stage IV & 0 & 0 & $25(58.1 \%)$ & 0 & 0 & $9(81.8 \%)$ & \\
\hline \multicolumn{8}{|l|}{ Braak Score } \\
\hline 0 & $15(36.6 \%)$ & $7(38.9 \%)$ & $1(2.3 \%)$ & 0 & 0 & 0 & \\
\hline$|-| \mid$ & $12(29.3 \%)$ & $5(27.8 \%)$ & $4(9.3 \%)$ & $6(26.1 \%)$ & 0 & 0 & \\
\hline III-IV & $14(34.1 \%)$ & $5(27.8 \%)$ & $31(72.1 \%)$ & $17(73.9 \%)$ & $7(12.5 \%)$ & $1(9.1 \%)$ & \\
\hline $\mathrm{V}-\mathrm{VI}$ & 0 & $1(5.6 \%)$ & $7(16.3 \%)$ & 0 & $49(87.5 \%)$ & $10(90.9 \%)$ & \\
\hline \multicolumn{8}{|l|}{ CERAD Score } \\
\hline 0 & $41(100 \%)$ & $14(77.8 \%)$ & $21(48.8 \%)$ & 0 & 0 & 0 & \\
\hline 1 & 0 & $4(22.2 \%)$ & $22(51.2 \%)$ & $22(95.7 \%)$ & $8(14.3 \%)$ & $1(9.1 \%)$ & \\
\hline 2 & 0 & 0 & 0 & $1(4.3 \%)$ & $26(46.4 \%)$ & $6(54.5 \%)$ & \\
\hline 3 & 0 & 0 & 0 & 0 & $22(39.3 \%)$ & $4(36.4 \%)$ & \\
\hline FTLD pathology & $10(27.7 \%)^{d, e}$ & $5(27.8 \%)^{d, e}$ & $6(14.6 \%)$ & $0(0 \%)^{a, b}$ & $3(5.4 \%)^{a, b}$ & $2(18.2 \%)$ & $<.05$ \\
\hline Tau & $9(25 \%)$ & $5(27.8 \%)$ & $6(14.6 \%)$ & 0 & $2(4.3 \%)$ & $2(18.2 \%)$ & \\
\hline TDP 43 & $1(2.7 \%)$ & $5(27.8 \%)$ & $4(9.8 \%)$ & 0 & $2(4.3 \%)$ & $1(9 \%)$ & \\
\hline$n$ total & 36 & 18 & 41 & 15 & 46 & 11 & \\
\hline LBD pathology & & & & & & & 0.31 \\
\hline Brainstem & $2(4.4 \%)$ & $1(5.6 \%)$ & $6(14 \%)$ & $2(13.3 \%)$ & $2(4.34 \%)$ & $0(0 \%)$ & \\
\hline Limbic/neocortical & $4(9.8 \%)$ & $2(11.1 \%)$ & $10(23.3 \%)$ & $1(6.6 \%)$ & $8(17.4 \%)$ & $4(36.4 \%)$ & \\
\hline$n$ & 36 & 18 & 43 & 15 & 46 & 11 & \\
\hline \multirow[t]{3}{*}{ Contact sports play } & Yes 12 (29.2\%) & Yes 17 (94.4\%) & Yes 43 (100\%) & Yes 0 & Yes $9(16 \%)$ & Yes 10 (90\%) & .013 \\
\hline & No $2(4.8 \%)$ & No 0 & No 0 & No $1(4 \%)$ & No 1 (1.7\%) & No NA & \\
\hline & Missing 27 (70.7\%) & Missing 1 (5.6\%) & Missing 0 & Missing $22(96 \%)$ & Missing $46(82 \%)$ & Missing 1 (9.1\%) & \\
\hline $\begin{array}{l}\text { CSF hemoglobin } \\
\text { (S.E.M.) }\end{array}$ & $481.0(106.6)$ & $648.3(261.2)$ & $465.1(106.5)$ & $555.6(183.6)$ & $386.5(93.1)$ & $370.5(192.7)$ & 0.85 \\
\hline$n$ & 36 & 11 & 33 & 23 & 49 & 11 & \\
\hline $\begin{array}{l}\text { Postmortem interval } \\
\text { (hours) (S.E.M) } 95 \% \mathrm{Cl}\end{array}$ & $\begin{array}{l}23.2(3.15)^{b} \\
16.82-29.56\end{array}$ & $\begin{array}{l}46.6(6.34)^{\mathrm{a}, \mathrm{d}, \mathrm{e}} \\
33.2-59.99\end{array}$ & $\begin{array}{l}33.88(3.02)^{\mathrm{e}} \\
27.77-39.98\end{array}$ & $\begin{array}{l}21.40(3.85)^{b} \\
13.43-29.37\end{array}$ & $\begin{array}{l}20.70(2.31)^{\mathrm{b}, \mathrm{c}} \\
16.1-25.32\end{array}$ & 24.99 (4.05) 15.98-34.0 & $<.001$ \\
\hline$N$ & 40 & 18 & 42 & 23 & 56 & 11 & \\
\hline PMI range (Max-Min) & $80.5 .00-1.75$ & $101.00-5.5$ & $96.00-2.00$ & $96.00-5.83$ & $99.15-1.75$ & $48.00-3.00$ & \\
\hline RIN (S.E.M.) $n$ & $6.2(0.2) 35$ & $5.76(0.5) 16$ & $5.6(0.2) 31$ & $5.7(0.5) 12$ & $5.8(0.2) 39$ & $4.7(0.4) 10$ & 0.15 \\
\hline pH (S.E.M.) $n$ & $6.14(0.08) 26$ & $6.14(0.10) 14$ & $6.17(0.1) 24$ & $6.07(0.13) 11$ & $6.08(0.06) 36$ & $5.97(0.8) 6$ & 0.83 \\
\hline
\end{tabular}

Data are presented mean with standard error of the (S.E.M.), years for age at death, and contact sports exposure and as \#yes/\#no (\%) unless otherwise indicated CERAD plaque density was rated as none (0), sparse (1), moderate (2), or frequent (3) for neuritic plaques

Int/High intermediate/high, AD Alzheimer disease, CERAD Consortium to Establish a Registry for Alzheimer's disease, CTE chronic traumatic encephalopathy, FTLD frontotemporal lobar degeneration, $L B D$ Lewy body disease, RIN RNA integrity number

${ }^{a}$ Different from no CTE/no AD ( $p<.05$, Bonferroni corrected)

${ }^{\mathrm{b}}$ Different from low CTE $(p<.05$, Bonferroni corrected)

${ }^{\mathrm{C}}$ Different from high CTE $(p<.05$, Bonferroni corrected)

${ }^{\mathrm{d}}$ Different from low $\mathrm{AD}(p<.05$, Bonferroni corrected)

e Different from intermediate/high $\mathrm{AD}(p<.05$, Bonferroni corrected)

${ }^{f}$ Different from CTE $+A D(p<.05$, Bonferroni corrected)

\# ANOVA with Bonferroni correction

${ }^{*} \chi^{2}$ test for proportions between all pathology groups 
pathologic groups. CSF analyte levels were used in a Kruskal-Wallis test performed to compare levels of analytes between pathologic groups. CSF measures were also rank-normalized (supplementary Figures e-1 and e-2) and used in one-way ANCOVAs in order to correct for age as a covariate to compare relative amounts of biomarkers between groups (supplementary results).

Statistical significance was set to $p<0.05$ following adjustments for multiple comparisons for all planned analyses. Binary logistic regression analyses were used to determine association between $\mathrm{p}$-tau $\mathrm{t}_{231}$ and $\mathrm{A} \beta_{1-42}$ and CTE or $\mathrm{AD}$ pathologic diagnosis controlling for age, sex, PMI, and other variables where appropriate. Linear regressions were performed to determine the relationship between CSF and brain $A \beta_{1-42}$ levels in AD and CTE. Receiver operating characteristic (ROC) curve analysis was used to determine sensitivity and specificity of CSF analytes between diagnoses.

\section{Results}

\section{Study population}

Participants were grouped based on the presence or absence of CTE and/or AD pathology. Group demographic differences for age at death, post-mortem interval (PMI), sex, RNA integrity number (RIN), $\mathrm{pH}$, and presence/absence of FTLD and LBD pathologies are listed in Table 1. Pathologic groups differed in age at death $(p<0.001)$ and PMI $(p<0.001)$ (Table 1). The low CTE group $(M=64.6 \pm 4.6)$ was younger than the low $\mathrm{AD}(M=86.7 \pm 1.1, p<.05)$, intermediate/high $\mathrm{AD}(M=$ $81.1 \pm 1.3, p<0.05)$, and CTE $+\mathrm{AD}$ groups $(M=79.6 \pm$ $2.8, p<0.05)$. The low $\mathrm{AD}$ group was older than the high CTE and control groups. The low CTE group had significantly longer PMIs $(M=46.6 \pm 6.4)$ than the control $(M=23.2 \pm 3.15 p<0.001)$, low $\mathrm{AD}(M=21.4 \pm 3.85, p$ $<0.001)$, and intermediate/high $\mathrm{AD}$ group $(M=20.7 \pm 2.3$, $p<0.05)$. The high CTE group $(M=33.88 \pm 3.02)$ had significantly longer PMIs than the intermediate/high AD group $(M=20.7 \pm 2.3, p<0.05)$. Despite these differences in PMI, there was no difference in RIN, $\mathrm{pH}$, or CSF hemoglobin between groups. A majority of men were present in each pathological group for all groups except for the intermediate/high AD group which had a majority of women. Years of contact sports differed between groups $(p=0.013)$ for the subset of participants that had this history taken $(n=95)$; post hoc pairwise comparisons revealed the control group $(M=9.46 \pm 2.97)$ had decreased years of contact sports compared to mild CTE group ( $M=20.85 \pm 3.43, p=0.036$ Bonferroni-corrected).

\section{All groups}

Comparing all pathological groups using the KruskalWallis test, there were significant differences in p-tau ${ }_{231}$
$\mathrm{H}(5)=16.28, p=0.006$ and $\mathrm{A} \beta_{1-42} \mathrm{H}(5)=19.32, p=0.002$. The low CTE group had significantly higher amount of p-tau ${ }_{231}$ (mean rank=121.1) compared to the Int/high $\mathrm{AD}$ group (mean rank $=74.2, p=0.014$ ). Both the high CTE group (mean rank $=58.74$ ) and low CTE (mean rank $=58.14$ ) groups were found to have significantly lower levels of $\mathrm{A} \beta_{1-42}$ compared to no CTE/no $\mathrm{AD}$ (control) (mean rank $=102.1, p<0.002$ and $p<0.05$, respectively). There was no significant difference in relative amounts of $\mathrm{p}$-tau ${ }_{181}$, total tau, and $\mathrm{A} \beta_{1-40}$ between all groups.

\section{Early stage disease}

Comparing no CTE/no AD (control), low CTE, and low $\mathrm{AD}$ pathological groups using the Kruskal-Wallis test, a difference in $\mathrm{p}-\operatorname{tau}_{231} \mathrm{H}(2)=8.93, p=0.015$ and $\mathrm{A} \beta_{1-42}$ $\mathrm{H}(2)=10.27, p=0.006$ was found. The low CTE group (mean rank $=52.61)$ had significantly higher levels of p-tau ${ }_{231}$ versus the no CTE/no AD (control) group (mean rank $=35.93, p=0.03$ ) and compared to the low $\mathrm{AD}$ group (mean rank $=33.02, p=0.017$ ) (Fig. 1). The low CTE group (mean rank 22.18) was also found to have significantly lower levels of $A \beta_{1-42}$ compared to the no CTE/no $\mathrm{AD}$ (control) (mean rank=41.51, $p=0.006$ ) (Fig. 1). The low $\mathrm{AD}$ group had lower levels of $\mathrm{A} \beta_{1-42}$ compared to the no CTE/no AD (control) group, but this difference was not significant $(p=0.19)$. There was no significant difference in relative amount of $\mathrm{p}$-tau ${ }_{181}$, total tau, or $A \beta_{1-40}$ between the no CTE/no AD (control), low CTE, and low AD groups.

Similar results for between group difference for early disease stages were obtained by rank-normalizing CSF measures (supplementary Figure e-1), followed by oneway ANCOVAs in order to correct for age as a covariate (supplementary results).

\section{Late stage disease}

Comparing high $\mathrm{CTE}, \mathrm{AD}$, and $\mathrm{CTE}+\mathrm{AD}$ pathological groups using the Kruskal-Wallis test, a difference in $\mathrm{p}-\mathrm{tau}_{231} \mathrm{H}(2)=7.096, p=0.029$ and $\mathrm{A} \beta_{1-42}$ $\mathrm{H}(2)=7.98, p=0.019$ was found overall. The High CTE group had significantly higher levels of $\mathrm{p}-\mathrm{tau}_{231}$ (mean rank $=59.61)$ compared to intermediate $/$ high $\mathrm{AD}$ (mean rank=43.3, $p=0.024$ ) (Fig. 2). The High CTE group also had significantly lower levels of $A \beta_{1}$. ${ }_{42}$ (mean rank $=36.34$ ) compared to the intermediate/high $\mathrm{AD}$ group (mean rank $=52.99, p=0.015$ ) (Fig. 2). There was no significant difference in relative amount of $\mathrm{p}-\operatorname{tau}_{181}$, total tau, or $\mathrm{A} \beta_{1-40}$ between High CTE, AD, and CTE + AD groups. Although there was no significant difference between groups for $\mathrm{ptau}_{181}$, all late stage group levels were all numerically greater than the control, indicating that ptau ${ }_{181}$ was elevated 


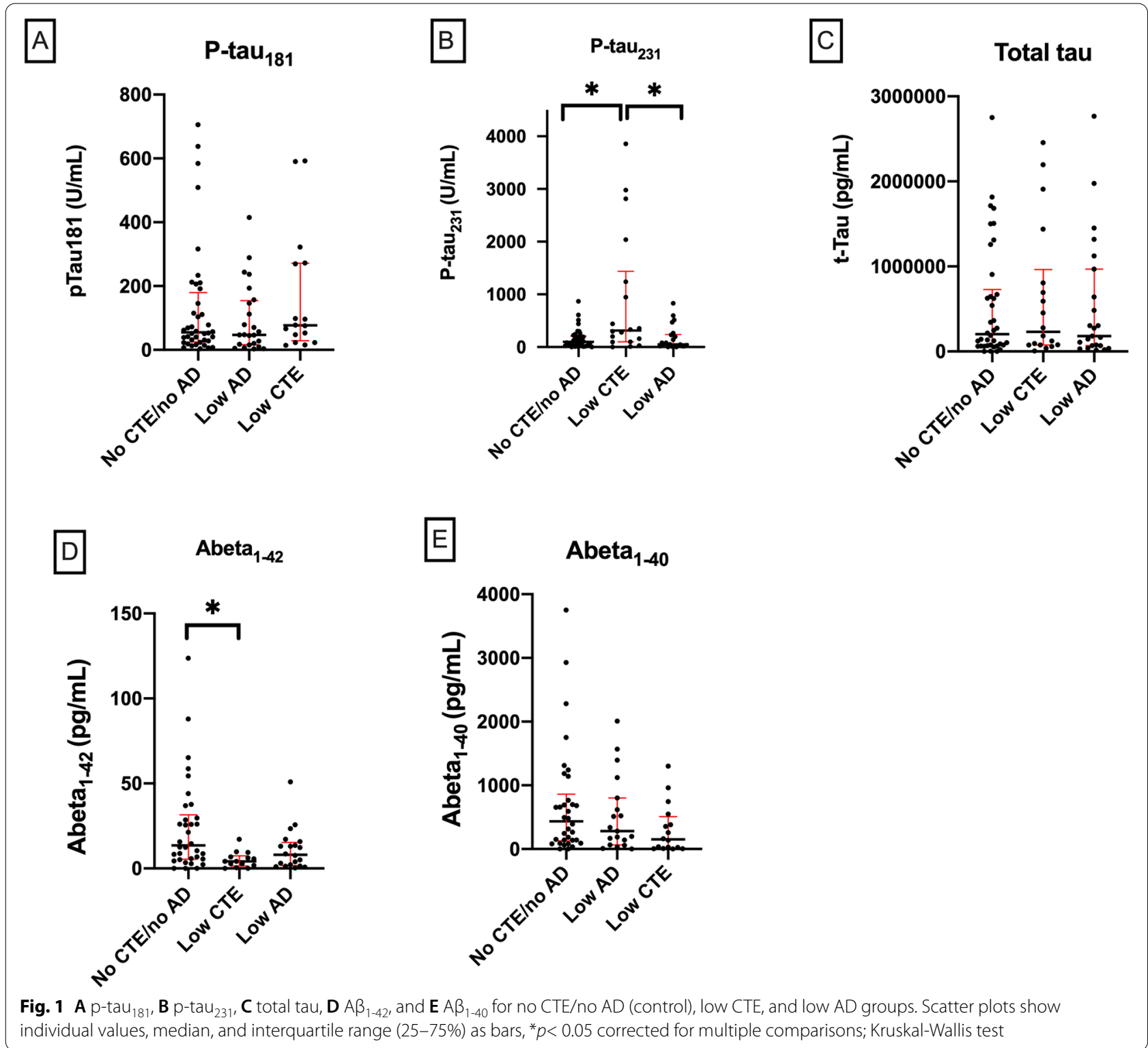

in late stage disease above the control group's levels as expected.

Results for between group difference for late disease stages were also obtained by rank-normalizing CSF measures (supplementary Figure e-2), followed by oneway ANCOVAs in order to correct for age as a covariate (supplementary results).

\section{Regression analyses}

We performed a binary logistic regression analysis to determine the contributions of $\mathrm{p}-\mathrm{tau}_{231}$ and $\mathrm{A} \beta_{1-42}$ in predicting pathological diagnosis of CTE (low and high CTE combined, excluding CTE +AD cases) vs. control. Variables included in the model were p-tau ${ }_{231}$,
$\mathrm{A} \beta_{1-42}$, age at death, and sex $(n=77)$. Both $\mathrm{p}-\operatorname{tau}_{231}$ (OR 1.53, 95\% CI 1.08-2.16) and $A \beta_{1-42}$ (OR 0.35, 95\% CI 0.17-0.74) were significant predictors of CTE status while controlling for age and sex, neither of which were significant predictors (Table 2). Secondary analyses including PMI, pH, and presence of LBD pathology as additional variables did not substantially change the results and were not significant predictors of CTE diagnosis. Although RIN and FTLD were associated with CTE, their presence in the model did not change the associations between ptau ${ }_{231}, \mathrm{~A} \beta_{1-42}$, and CTE status.

We also performed a binary logistic regression to determine the contributions of $\mathrm{p}-\mathrm{tau}_{231}$ and $\mathrm{A} \beta_{1-42}$ in predicting pathological diagnosis of CTE (low and high 


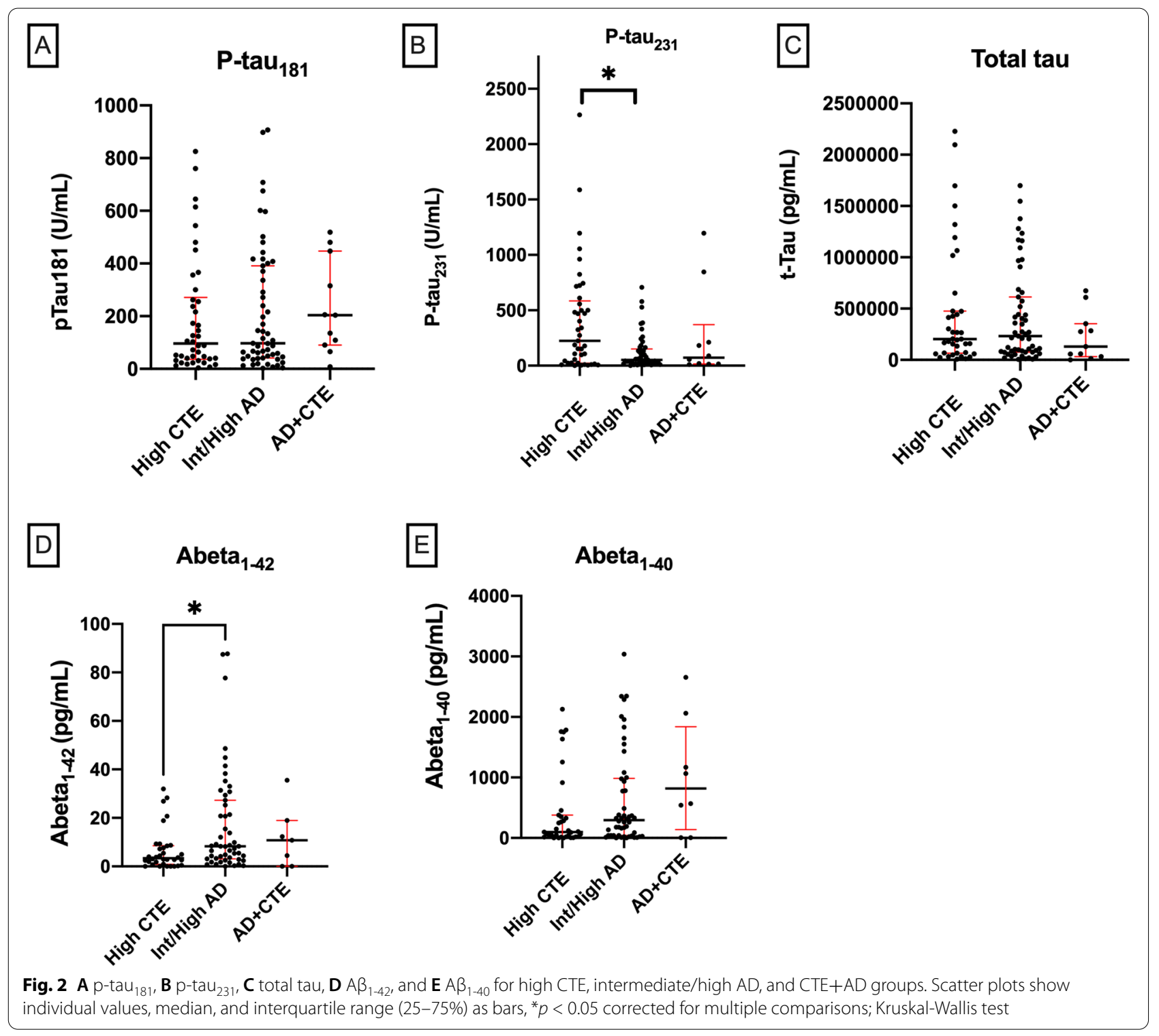

CTE combined) vs. AD (low $\mathrm{AD}$ and intermediate/high AD combined) (Table 3). Variables included in the model were $\mathrm{p}-\mathrm{tau}_{231}, \mathrm{~A} \beta_{1-42}$, age at death, and sex $(n=111)$. Both p-tau ${ }_{231}$ (OR 1.34, 95\% CI 1.02-1.76, $p=0.036$ ) and $\mathrm{A} \beta_{1-42}$ (OR 0.51, 95\% CI 0.28-0.91) were found to distinguish between CTE and AD diagnoses, controlling for age at death (OR 0.91, 95\% CI 0.84-0.97) and sex which was not a significant predictor. A secondary analysis adjusting for PMI $(n=102)$, demonstrated that this trend continued but with a decrease in significance most likely due to decreased power, with p-tau ${ }_{231}$ (OR 1.23, 95\% CI 0.90-1.72), $\mathrm{A} \beta_{1-42}$ (OR 0.60, 95\% CI 0.31-1.15), adjusting for age (OR 0.88, 95\% CI 0.81-0.97), PMI (OR 1.06, 95\% CI 1.01-1.12), and sex, which was not a significant
Table 2 CSF predictors of CTE pathology versus no CTE/no AD (control)

\begin{tabular}{llll}
\hline & OR & $\begin{array}{l}\text { 95\% Confidence } \\
\text { Interval }\end{array}$ & $\boldsymbol{p}$ value \\
\hline$p-\operatorname{tau}_{231}(\mathrm{U} / 10 \mu \mathrm{L})$ & 1.53 & $1.08-2.16$ & 0.016 \\
$\mathrm{~A} \beta_{1-42}(\mathrm{pg} / 100 \mu \mathrm{L})$ & 0.35 & $0.17-0.74$ & 0.006 \\
\hline
\end{tabular}

Binary logistic regression comparing CTE all stages with no CTE/no AD (control) group while adjusting for age and sex $(n=77)$

predictor. Additional secondary analyses included the addition of RIN and pH separately as well as presence/ absence of FTLD pathology and LBD pathology, none of which were significant predictors of CTE diagnosis. 
Table 3 CSF predictors of CTE versus AD pathology

\begin{tabular}{llll}
\hline & OR & $\begin{array}{l}\text { 95\% confidence } \\
\text { interval }\end{array}$ & $\boldsymbol{p}$ value \\
\hline $\mathrm{p}-\mathrm{tau}_{231}(\mathrm{U} / 10 \mu \mathrm{L})$ & 1.34 & $1.02-1.76$ & 0.036 \\
$\mathrm{~A} \beta_{1-42}(\mathrm{pg} / 100 \mu \mathrm{L})$ & 0.51 & $0.28-0.91$ & 0.022 \\
Age of death & 0.91 & $0.84-0.97$ & 0.006 \\
\hline
\end{tabular}

Binary logistic regression comparing CTE all stages with $A D$ all stages adjusting for age and sex $(n=111)$ predictors of cortical $A \beta_{1-42}$ levels while age was found to be a significant predictor $(\beta=0.38, p=0.001)$.

\section{Receiver Operating Characteristic curve (ROC) analyses}

To assess the diagnostic accuracy of CSF p-tau 231 and $\mathrm{A} \beta_{1-42}$ levels, an ROC analysis was performed to determine if $\mathrm{p}-\mathrm{tau}_{231}, \mathrm{~A} \beta_{1-42}$, age at death, and sex were predictive of CTE vs. control diagnosis. Area under the curve (AUC) was 0.88 (SEM $=0.04, p<0.001)$ (Fig. 3A). A separate $\mathrm{ROC}$ analysis was performed for CTE vs. AD diag-

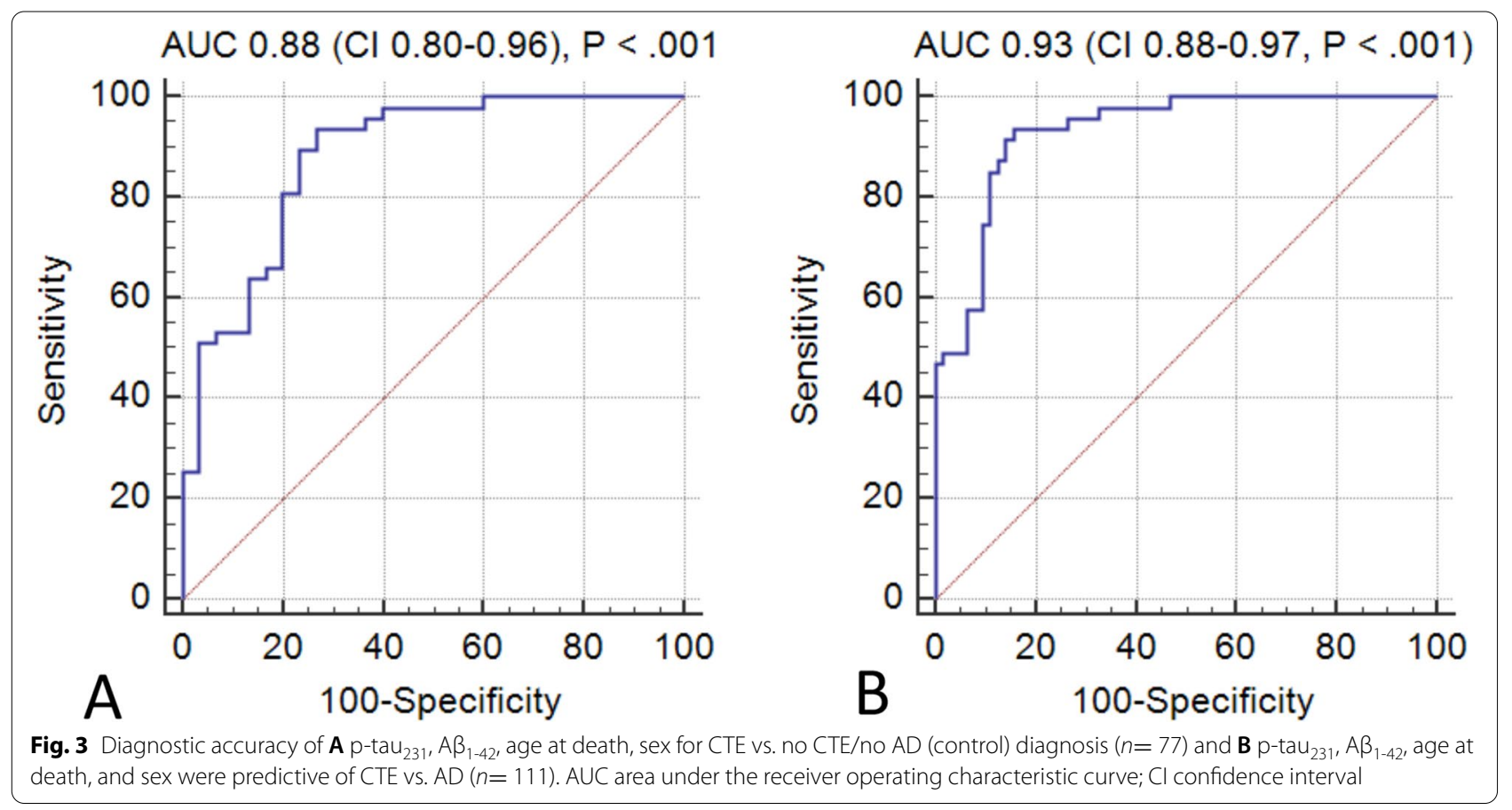

Given the surprisingly low levels of $A \beta_{1-42}$ in CTE, we performed a secondary analysis to test the hypothesis CSF beta-amyloid levels reflect brain tissue $A \beta_{1-42}$ levels in $\mathrm{AD}$, but not in CTE. Using a linear regression it was found that a model including age, sex, and CSF $\mathrm{A} \beta_{1-42}$ levels predicted a significant amount of the variance of brain $A \beta_{1-42}$ levels among the $A D$ and control groups combined $\left(\mathrm{F}(3,100)=5.42, p=0.002, R^{2}=0.14\right.$, adjusted $\left.R^{2}=0.114\right)$ and both CSF $\mathrm{A} \beta_{1-42}(\beta=-0.21$, $p=0.032)$ and age $(\beta=0.21, p=0.036)$ predicted frontal cortex $\mathrm{A} \beta_{1-42}$ while sex $(\beta=0.13, p=0.19)$ did not. In a separate linear regression among the combined CTE and control groups, the overall regression model including CSF $A \beta_{1-42}$ levels, age, and sex predicted a significant amount of the variance of brain $A \beta_{1-42}$ levels $\left(\mathrm{F}(3,77)=4.52, p=0.006, R^{2}=0.15\right.$, adjusted $\left.R^{2}=.117\right)$, though CSF A $\beta_{1-42}$ levels $(\beta=-0.17, p=0.11)$ and sex $(\beta=-0.14, p=0.21)$ were not found to be a significant nosis to determine if $\mathrm{p}$-tau $\mathrm{tan}_{23}, \mathrm{~A} \beta_{1-42}$, age at death, and sex were predictive and demonstrated that AUC was 0.93 $(\mathrm{SEM}=0.023, p<0.001)$ (Fig. 3B).

\section{Discussion}

In summary, our results indicate that in post-mortem CSF, p-tau 231 levels were significantly higher in both the low and high CTE groups compared to the no CTE/no $\mathrm{AD}$ (control) group and compared to the AD groups. Furthermore, surprisingly CSF $A \beta_{1-42}$ was found to be decreased in both low stage CTE compared to the no $\mathrm{CTE} /$ no $\mathrm{AD}$ (control) group as well as in high stage CTE compared to Int/high AD. Overall, increased levels of p-tau ${ }_{231}$ and decreased levels of $A \beta_{1-42}$ in combination were found to be significant predictors of CTE compared to controls and AD. 
While three repeat and four repeat tau species are present in both $\mathrm{AD}$ and CTE [22, 23], unique folding patterns of tau have been reported to predominate in CTE as compared to AD [24]. Beyond a limited number of studies, little is known regarding the exact tau species present in CTE and how they can be distinguished at the epitope level from the tauopathy of $\mathrm{AD}$, though we recently found that both $\mathrm{ptau}_{202}$ and p-tau ${ }_{231}$ are associated with increased years of RHI from American football [25]. Increased p-tau ${ }_{231}$ levels in CTE may be due to tau leakage from cells into the CSF, which may be present even relatively early on in the disease course when clinical symptoms are relatively mild. P-tau ${ }_{231}$ was significantly increased in low CTE, and there was a trend towards an increase for high stage CTE groups compared to AD groups, which could reflect increased neuron and axonal damage in CTE. Studies in AD have revealed that increased phosphorylated tau levels, including both $\mathrm{p}$ - $\operatorname{tau}_{181}$ and $\mathrm{p}$-tau $\mathrm{tan}_{231}$, correlate with neocortical neurofibrillary burden in $\mathrm{AD}[26,27]$, though a similar relationship in CTE between elevated CSF p-tau 231 levels and increased p-tau accumulation in the neocortex has not been well-investigated. While t-tau may be a more general marker of neurodegeneration reflecting axonal injury, CSF p-tau ${ }_{181}$ and p-tau ${ }_{231}$ may be specific for the intraneuronal tau pathology seen in both CTE and AD. Our findings from the current study suggest that $\mathrm{p}-\mathrm{tau}_{231}$ CSF levels may be more sensitive and specific than both $\mathrm{t}$-tau and $\mathrm{p}$ - $\mathrm{tau}_{181}$ in distinguishing CTE from non-CTE and non-AD participants as well as in distinguishing CTE from $\mathrm{AD}$.

The role of $A \beta$ accumulation in CTE pathophysiology is not well understood. In the current study, the low CTE group exhibited lower $A \beta_{1-42}$ levels in post-mortem CSF compared to those in the control group and surprisingly the High CTE group had decreased $A \beta_{1-42}$ levels compared to the intermediate/high AD group. Decreased CSF $A \beta_{1-42}$ in combination with elevations in tau species and relatively unchanged $A \beta_{1-40}$ are well-established markers of $\mathrm{AD}$ and predict the conversion of mild cognitive impairment (MCI) to AD [28-30]. In vivo studies of former NFL players with objective memory deficits have not been found to have increased $A \beta$ deposition compared to controls as measured by florbetapir PET, indicating that cognitive decline in former players at risk of possible CTE was not related to $A D$ or $A \beta$ deposition [31]. In contrast, post-mortem evidence has shown that $\mathrm{A} \beta$ accumulation can occur in CTE; however, it is not a consistent pathologic feature and is absent in about half of cases, tending to form diffuse rather than neuritic plaques when present [11]. However, there does appear to be an age-dependent acceleration of $A \beta$ deposition in CTE compared to a normal autopsy population [11].
Furthermore, RHI associated with CTE likely damages blood vessels and is associated with the development of frontal leptomeningeal cerebral amyloid angiopathy (CAA) which is distinct from $\mathrm{AD}$ [32]. This preferential localization of $A \beta$ associated with CAA in the leptomeningeal vessels suggests that the mechanism of decreased $A \beta_{1-42}$ seen in CTE may be related to impaired CSF clearance of $A \beta$, rather than to amyloid sequestration in plaques. We found that there was a significant relationship between CSF and brain levels of $A \beta_{1-42}$ in AD, but not between CSF and brain levels in CTE. This may indicate that sequestration of $A \beta_{1-42}$ in plaques may drive CSF levels of $A \beta_{1-42}$ in $A D$, but that CSF $A \beta_{1-42}$ is determined by a different mechanism in CTE.

The relationship of the two pathologies underlying $\mathrm{AD}$ and CTE is not well understood and as a starting point this study has sought to investigate the levels of several CSF AD biomarkers in the CTE population with the general hypothesis that alterations in these analytes would allow improved discrimination of $\mathrm{AD}$ and CTE. Older individuals with CTE pathology are more likely to have concurrent amyloid-beta $(\mathrm{A} \beta)$ plaques consistent with $A D[16]$ and may also develop $A \beta$ accumulation at a younger age than individuals without head injuries [11]. In prior studies $13 \%$ of neuropathologically confirmed CTE cases also had AD pathology [33]. In cases where CTE co-occurs with AD, it is unclear if individuals developed a mixed pathology neurodegenerative disorder or whether there is a synergistic relationship between the two pathophysiological processes. An additional possibility is that decreases in CSF A $\beta$ may not correlate as closely with increased $A \beta$ plaque deposition in CTE, a potential departure from the well-established inverse correlation between CSF $A \beta$ levels and $A \beta$ plaque burden in $\mathrm{AD}$. Although we did not find overall group differences in $\mathrm{p}$ - $\mathrm{tau}_{181}$ and $\mathrm{t}$-tau between any of the pathologic groups, the intermediate/severe AD did show the expected increases in $\mathrm{p}$-tau ${ }_{181}$ and $\mathrm{t}$-tau compared to the no $\mathrm{AD} /$ no CTE group.

When controlling for age and sex, both $\mathrm{p}-\mathrm{tau}_{231}$ and $A \beta_{1-42}$ were able to discriminate CTE from controls using binary logistic regressions. $p-\operatorname{tau}_{231}$ and $A \beta_{1-42}$ were still significant when controlling for PMI, RIN, or $\mathrm{pH}$ individually. PMI, RIN, and $\mathrm{pH}$ all generally correlate with one another. $\mathrm{p}-\mathrm{tau}_{231}$ and $\mathrm{A} \beta_{1-42}$ were also able to discriminate CTE from AD when controlling for age and sex, but lost significance when controlling for PMI, possibly related to decreased power.

\section{Limitations}

This study is limited in that age is a major risk factor for neurodegenerative conditions and the CTE groups were younger than the AD groups. As a result, age at death 
was included in the regression analysis but there is still the possibility that age could act as a potential confounder in the current study. Differences in years of play generally differ by diagnosis with CTE groups having increased years of play exposure; however, this variable was missing in a significant percentage of cases and as such contact sports play is a potential confounder that could not be accounted for in our models. Furthermore, the groups did not have individuals of each gender evenly distributed and the AD group had increased numbers of women compared to the CTE groups which did not have women. This is a concern as gender differences in neuropathology have previously been reported [34]. However, we have attempted to correct for this in part by running regression and sensitivity analyses among men only and have found similar results for both ptau ${ }_{231}$ and $A \beta_{1-42}$ compared to analyses run with both men and women. In addition, this study was autopsy-based and thus potentially subject to selection bias as individuals whose brains are donated by family may not represent the population more broadly. However, grouping by known pathology allows for definitive associations not possible in a clinical sample where the pathology is unknown. The current study was also limited in that it is post-mortem, and CSF abeta and total tau levels are known to be altered from antemortem levels in post-mortem samples [35]. Both the low and high CTE groups had significantly longer PMIs than the no CTE/no AD (control), low AD, and intermediate/high $\mathrm{AD}$ groups which raises concern that a longer PMI could lead to increased tau levels in the CTE groups. We attempted to take this into account by controlling for PMI when possible. Furthermore, given its post-mortem nature, the current study serves not to set a definitive cut-off but to warrant ante-mortem comparison of these amyloid and tau levels in CSF among patients with suspected CTE and AD. Of note, t-tau, $\mathrm{p}$-tau ${ }_{181}$, and $\mathrm{A} \beta_{42}$ have been previously evaluated during life in former professional American football players, at increased risk of CTE, and it was found that increased cumulative head impact exposure in former players predicted t-tau levels [36].

Several promising modalities have been investigated for their use as in vivo CTE biomarkers including PET imaging of p-tau [31], peripheral blood levels of total tau and exosomal tau [37, 38], and CSF biomarkers [39, 40]. CSF biomarkers are particularly promising as they closely reflect the dynamic relationship of solute clearance in the glymphatic space [41] and thus may provide a window to relatively early neuropathological changes. Future studies should investigate other tau isoforms that have recently shown promise in $\mathrm{AD}$ including $\mathrm{p}$ - $\mathrm{tau}_{217}[42]$ as well as plasma biomarkers of tau isoforms [7, 43, 44].

\section{Conclusions}

Overall, the current study revealed that levels of postmortem CSF $\mathrm{p}-\mathrm{tau}_{231}$ and $\mathrm{A} \beta_{1-42}$ were selectively altered in groups with CTE compared to those with $\mathrm{AD}$, and compared to those without $\mathrm{CTE}$ and without $\mathrm{AD}$. Both $\mathrm{p}-\mathrm{tau}_{231}$ and $\mathrm{A} \beta_{1-42}$ were also predictive of CTE diagnosis compared to AD group and compared to non-CTE/non$\mathrm{AD}$ groups, indicating that $\mathrm{p}-\mathrm{tau}_{231}$ represents a potentially sensitive and specific biomarker of CTE, and that decreases in CSF $A \beta_{1-42}$ should be further investigated in vivo among possible CTE patients.

\section{Abbreviations}

CSF: Cerebrospinal fluid; CTE: Chronic traumatic encephalopathy; AD: Alzheimer's disease; Aß: Amyloid-beta; t-tau: Total tau; p-tau: Phosphorylated tau; VA-BU-CLF: Veteran's Affairs-Boston University-Concussion Legacy Foundation Brain Bank; UNITE: Understanding Neurologic Injury and Traumatic Encephalopathy; BU ADRC: Boston University's Alzheimer's Disease Research Center; NACC): National Alzheimer's Disease Coordinating Center; UDS: Uniform Data Set; FHS: Framingham Heart Study; RHI: Repetitive head impact; LBD: Lewy body disease; FTLD: Frontotemporal lobar degeneration; ALS/MND: Amyotrophic Lateral Sclerosis/motor neuron disease; ANOVA: One-way analysis of variance; PMI: Post-mortem interval; RIN: RNA integrity number; ROC: Receiver Operating Characteristic curve; AUC: Area under the curve; MCI: Mild cognitive impairment; CAA: Cerebral amyloid angiopathy.

\section{Supplementary Information}

The online version contains supplementary material available at https://doi. org/10.1186/s13195-022-00976-y.

Additional file 1. Supplemental results

Additional file 2: Figure e-1. Rank-normalized fold change of A. p-tau 181 , B. p-tau ${ }_{231}$, C. total tau, D. $A \beta_{1-42}$ and E. $A \beta_{1-40}$ for no CTE/no AD (control), Low CTE, and Low AD groups. Scatter plots show individual values, median and interquartile range (25-75\%) as bars, ${ }^{*} p<0.05$ corrected for multiple comparisons; ANCOVA adjusting for age.

Additional file 3: Figure e-2. Rank-normalized fold change of A. $p$-tau ${ }_{181}$, B. p-tau ${ }_{231}$, C. total tau, D. $A \beta_{1-42}$ and E. $A \beta_{1-40}$ for High CTE, Intermediate/High $A D$, and $C T E+A D$ groups. Scatter plots show individual values, median and interquartile range (25-75\%), ${ }^{*} p<0.05$ corrected for multiple comparisons; ANCOVA adjusting for age.

Additional file 4: Table e-1. Estimated Marginal Means and SEM in parentheses for rank normalized CSF analyte measurements from No CTE/no AD (control) group; showing ANCOVA adjusted for age, $p<0.05$. Table e-2. Estimated Marginal Means and SEM in parentheses for rank normalized CSF analyte measurements from No CTE/no AD (control) group; showing ANCOVA adjusted for age, sex, and PMI, $p<0.05$.

\section{Acknowledgements}

We gratefully acknowledge the use of resources and facilities at the Edith Nourse Rogers Memorial Veterans Hospital (Bedford, MA) as well as all the individuals whose participation and contributions made this work possible.

\section{Authors' contributions}

KWT played a major role in drafting manuscript writing, revisions, and data analysis and interpretation. AG helped with the initial manuscript drafting and data entry and analysis. VEA helped with the data generation and provided a critical manuscript review for content. WX helped with the brain and CSF ELISA data generation and analysis, and critical manuscript review for content. JDC provided assistance with data interpretation, critical manuscript review for content. RN provided assistance with brain and CSF data generation and 
analyses. GM helped with brain and CSF ELISA data generation and analysis. SD helped with brain and CSF ELISA data generation. YT provided assistance with statistical plan, data generation, and critical manuscript review for content. BH helped with data generation, analyses, and critical manuscript review for content. AEB provided assistance with data analysis and critical manuscript review for content. BD provided assistance with data analysis and critical manuscript review for content. NK provided assistance with data analysis and critical manuscript review for content. RCC provided assistance with data analysis and critical manuscript review for content. LEG provided assistance with data analysis and critical manuscript review for content. DIK provided assistance with data analysis and critical manuscript review for content. RAS provided assistance with data analysis and critical manuscript review for content. MLA provided assistance with data analysis and critical manuscript review for content. JM provided assistance with statistical plan, data analysis, and critical manuscript review for content. ACM provided assistance with data analysis and critical manuscript review for content. TDS led all aspects of study including concept and design, data analysis and interpretation, and critical manuscript writing and editing. The author(s) read and approved the final manuscript.

\section{Funding}

This work was supported by the United States (U.S.) Department of Veterans Affairs, Veterans Health Administration, Clinical Sciences Research and Development Merit Awards (101-CX001038) (CX001698-01A2); Career Development Award-2 (\# IK2 CX002065) Alzheimer's Association (NIRG-305779, NIRG-362697); National Institute of Aging (RF1AG054156, R56AG057768, RF1AG057768, K23AG046377, U19AG068753, AG08122, AG054076); National Institute of Neurological Disorders and Stroke (U54NS1 15266, U01NS086659, K23NS102399); National Institute of Aging Boston University AD Center (P30-AG072978; supplement 0572063345-5; pilot award 50204517); National Heart, Lung and Blood Institute (75N92019D00031 and HHSN2682015000011); Department of Defense Peer Reviewed Alzheimer's Research Program (PRARP \#13267017); and the Concussion Legacy Foundation. This work was also supported by unrestricted gifts from the Andlinger Foundation and WWE.

\section{Availability of data and materials}

Anonymized data not published within the article will be shared upon reasonable request.

\section{Declarations}

\section{Ethics approval and consent to participate}

All study protocols were approved by the VA Bedford Healthcare System and Boston University Medical Center Institutional Review Boards. Written and informed consent was obtained from all patients and family members participating.

\section{Consent for publication}

Not applicable.

\section{Competing interests}

Andrew E. Budson has been a speaker for Eli Lilly, a consultant for General Electric, Eli Lilly, and Axovant. Lee E. Goldstein has received funding from the WWE and Ivivi Health Sciences. Robert A. Stern is a member of the MackeyWhite Committee of the NFL Players Association. He is a paid consultant to BioGen (Cambridge, MA, USA) and Eli Lilly (Indianapolis, IN, USA). He receives royalties for published neuropsychological tests from Psychological Assessment Resources, Inc. (Lutz, FL, USA) and is a member of the Board of Directors of King-Devick Technologies (Chicago, IL, USA). Robert C. Cantu is a paid consultant to the NFL Head Neck and Spine Committee, NOCSAE, Concussion Legacy Foundation, receives royalties from book publications, and compensation from expert legal opinion. He is a member of the Mackey-White Committee of the NFL Players Association. Ann C. McKee is a member of the Mackey-White Committee of the NFL Players Association. The other authors have no competing interests or disclosures to report.

\section{Author details}

'Boston University Alzheimer's Disease Research and CTE Center, Boston University School of Medicine, Boston, MA 02118, USA. ${ }^{2}$ VA Boston Healthcare
System, 150 S. Huntington Avenue, Boston, MA 02130, USA. ${ }^{3}$ Department of Neurology, Boston University School of Medicine, Boston, MA 20118, USA. ${ }^{4}$ Boston University School of Medicine, Boston, MA 02118, USA. ${ }^{5}$ VA Bedford Healthcare System, Bedford, MA 01730, USA. ${ }^{6}$ Department of Biostatistics, Boston University School of Public Health, Boston, MA 20118, USA. ${ }^{7}$ Braintree Rehabilitation Hospital, Braintree, MA 02118, USA. ${ }^{8}$ Department of Anatomy and Neurobiology, Boston University School of Medicine, Boston, MA 20119, USA. ${ }^{9}$ Concussion Legacy Foundation, Boston, MA 02115, USA. ${ }^{10}$ Department of Neurosurgery, Boston University School of Medicine, Boston, MA 02118, USA. " ${ }^{12}$ Department of Neurosurgery, Emerson Hospital, Concord, MA 01742, USA. ${ }^{12}$ Departments of Psychiatry, Ophthalmology, Boston University School of Medicine, Boston, USA. ${ }^{13}$ Departments of Biomedical, Electrical \& Computer Engineering, Boston University College of Engineering, Boston, USA. ${ }^{14}$ Department of Pathology and Laboratory Medicine, Boston University School of Medicine, Boston, MA 02118, USA.

Received: 13 May 2021 Accepted: 2 February 2022

Published online: 09 February 2022

\section{References}

1. McKee AC, Cairns NJ, Dickson DW, Folkerth RD, Keene CD, Litvan I, et al. The first NINDS/NIBIB consensus meeting to define neuropathological criteria for the diagnosis of chronic traumatic encephalopathy. Acta Neuropathol. 2016;131(1):75-86.

2. Hof PR, Bouras C, Buee L, Delacourte A, Perl DP, Morrison JH. Differential distribution of neurofibrillary tangles in the cerebral cortex of dementia pugilistica and Alzheimer's disease cases. Acta Neuropathol. 1992;85(1):23-30.

3. Tokuda T, Ikeda S, Yanagisawa N, Ihara Y, Glenner GG. Re-examination of ex-boxers' brains using immunohistochemistry with antibodies to amyloid beta-protein and tau protein. Acta Neuropathol. 1991;82(4):280-5.

4. Braak H, Braak E. Staging of Alzheimer's disease-related neurofibrillary changes. Neurobiol Aging. 1995;16(3):271-8 discussion 8-84.

5. Katsumoto A, Takeuchi H, Tanaka F. Tau pathology in chronic traumatic encephalopathy and Alzheimer's disease: similarities and differences. Front Neurol. 2019;10:980

6. Schmidt ML, Zhukareva V, Newell KL, Lee VM, Trojanowski JQ. Tau isoform profile and phosphorylation state in dementia pugilistica recapitulate Alzheimer's disease. Acta Neuropathol. 2001;101(5):518-24.

7. Ashton NJ, Leuzy A, Karikari TK, Mattsson-Carlgren N, Dodich A, Boccardi $\mathrm{M}$, et al. The validation status of blood biomarkers of amyloid and phospho-tau assessed with the 5-phase development framework for AD biomarkers. Eur J Nucl Med Mol Imaging. 2021;48:2140-56.

8. Zetterberg H, Blennow K. Moving fluid biomarkers for Alzheimer's disease from research tools to routine clinical diagnostics. Mol Neurodegener. 2021;16(1):10.

9. McGrowder DA, Miller F, Vaz K, Nwokocha C, Wilson-Clarke C, AndersonCross M, et al. Cerebrospinal fluid biomarkers of Alzheimer's disease: current evidence and future perspectives. Brain Sci. 2021;11(2):215.

10. Ehrenberg AJ, Khatun A, Coomans E, Betts MJ, Capraro F, Thijssen EH, et al. Relevance of biomarkers across different neurodegenerative diseases. Alzheimers Res Ther. 2020;12(1):56.

11. Stein TD, Montenigro PH, Alvarez VE, Xia W, Crary JF, Tripodis Y, et al. Beta-amyloid deposition in chronic traumatic encephalopathy. Acta Neuropathol. 2015;130(1):21-34.

12. Alosco ML, Cherry JD, Huber BR, Tripodis Y, Baucom Z, Kowall NW, et al. Characterizing tau deposition in chronic traumatic encephalopathy (CTE): utility of the McKee CTE staging scheme. Acta Neuropathol. 2020;140(4):495-512.

13. Nelson PT, Jicha GA, Schmitt FA, Liu H, Davis DG, Mendiondo MS, et al. Clinicopathologic correlations in a large Alzheimer disease center autopsy cohort: neuritic plaques and neurofibrillary tangles "do count" when staging disease severity. J Neuropathol Exp Neurol. 2007;66(12):1136-46.

14. Mez J, Solomon TM, Daneshvar DH, Murphy L, Kiernan PT, Montenigro $\mathrm{PH}$, et al. Assessing clinicopathological correlation in chronic traumatic encephalopathy: rationale and methods for the UNITE study. Alzheimers Res Ther. 2015;7(1):62. 
15. Adams JW, Alvarez VE, Mez J, Huber BR, Tripodis Y, Xia W, et al. Lewy Body pathology and chronic traumatic encephalopathy associated with contact sports. J Neuropathol Exp Neurol. 2018;77(9):757-68.

16. Mckee AC, Stern RA, Nowinski CJ, Stein TD, Alvarez VE, Daneshvar DH, et al. The spectrum of disease in chronic traumatic encephalopathy. Brain. 2013;136(Pt 1):43-64.

17. Boyle PA, Yu L, Wilson RS, Leurgans SE, Schneider JA, Bennett DA. Personspecific contribution of neuropathologies to cognitive loss in old age. Ann Neurol. 2018:83(1):74-83.

18. Karanth S, Nelson PT, Katsumata Y, Kryscio RJ, Schmitt FA, Fardo DW, et al. Prevalence and clinical phenotype of quadruple misfolded proteins in older adults. JAMA Neurol. 2020;77(10):1299-307.

19. McKeith IG. Consensus guidelines for the clinical and pathologic diagnosis of dementia with Lewy bodies (DLB): report of the Consortium on DLB International Workshop. J Alzheimers Dis. 2006;9(3 Suppl):417-23.

20. Mackenzie IR, Neumann M, Bigio EH, Cairns NJ, Alafuzoff I, Kril J, et al. Nomenclature and nosology for neuropathologic subtypes of frontotemporal lobar degeneration: an update. Acta Neuropathol. 2010;119(1):1-4.

21. Cairns NJ, Bigio EH, Mackenzie IR, Neumann M, Lee VM, Hatanpaa KJ, et al. Neuropathologic diagnostic and nosologic criteria for frontotemporal lobar degeneration: consensus of the Consortium for Frontotemporal Lobar Degeneration. Acta Neuropathol. 2007;114(1):5-22.

22. Cherry JD, Esnault CD, Baucom ZH, Tripodis Y, Huber BR, Alvarez VE, et al. Tau isoforms are differentially expressed across the hippocampus in chronic traumatic encephalopathy and Alzheimer's disease. Acta Neuropathol Commun. 2021;9(1):86

23. Cherry JD, Kim SH, Stein TD, Pothast MJ, Nicks R, Meng G, et al. Evolution of neuronal and glial tau isoforms in chronic traumatic encephalopathy. Brain Pathol. 2020;30(5):913-25.

24. Falcon B, Zivanov J, Zhang W, Murzin AG, Garringer HJ, Vidal R, et al. Novel tau filament fold in chronic traumatic encephalopathy encloses hydrophobic molecules. Nature. 2019;568(7752):420-3.

25. Stathas S, Alvarez VE, Xia W, Nicks R, Meng G, Daley S, et al. Tau phosphorylation sites serine and serine are differently altered in chronic traumatic encephalopathy and Alzheimer's disease. Alzheimers Dement. 2021. https://doi.org/10.1002/alz.12502.

26. Buerger K, Ewers M, Pirttila T, Zinkowski R, Alafuzoff I, Teipel SJ, et al. CSF phosphorylated tau protein correlates with neocortical neurofibrillary pathology in Alzheimer's disease. Brain. 2006;129(Pt 11):3035-41.

27. Tapiola T, Alafuzoff I, Herukka SK, Parkkinen L, Hartikainen P, Soininen H, et al. Cerebrospinal fluid \{beta\}-amyloid 42 and tau proteins as biomarkers of Alzheimer-type pathologic changes in the brain. Arch Neurol. 2009;66(3):382-9.

28. Hampel H, Burger K, Teipel SJ, Bokde AL, Zetterberg H, Blennow K. Core candidate neurochemical and imaging biomarkers of Alzheimer's disease. Alzheimers Dement. 2008:4(1):38-48.

29. Hansson O, Zetterberg H, Buchhave P, Londos E, Blennow K, Minthon L. Association between CSF biomarkers and incipient Alzheimer's disease in patients with mild cognitive impairment: a follow-up study. Lancet Neurol. 2006;5(3):228-34.

30. Mattsson N, Zetterberg $\mathrm{H}$, Hansson $\mathrm{O}$, Andreasen $\mathrm{N}$, Parnetti $\mathrm{L}$, Jonsson $M$, et al. CSF biomarkers and incipient Alzheimer disease in patients with mild cognitive impairment. JAMA. 2009:302(4):385-93.

31. Stern RA, Adler CH, Chen K, Navitsky M, Luo J, Dodick DW, et al. Tau positron-emission tomography in former national football league players. N Engl J Med. 2019;380(18):1716-25.

32. Standring OJ, Friedberg J, Tripodis Y, Chua AS, Cherry JD, Alvarez VE, et al. Contact sport participation and chronic traumatic encephalopathy are associated with altered severity and distribution of cerebral amyloid angiopathy. Acta Neuropathol. 2019;138(3):401-13.

33. McKee AC, Stein TD, Kiernan PT, Alvarez VE. The neuropathology of chronic traumatic encephalopathy. Brain Pathol. 2015;25(3):350-64.

34. Mielke MM, Vemuri P, Rocca WA. Clinical epidemiology of Alzheimer's disease: assessing sex and gender differences. Clin Epidemiol. 2014;6:37-48.

35. Morihara T, Kudo T, Ikura Y, Kashiwagi Y, Miyamae Y, Nakamura Y, et al. Increased tau protein level in postmortem cerebrospinal fluid. Psychiatry Clin Neurosci. 1998;52(1):107-10.

36. Alosco ML, Tripodis Y, Fritts NG, Heslegrave A, Baugh CM, Conneely S, et al. Cerebrospinal fluid tau, Abeta, and sTREM2 in Former National Football League Players: modeling the relationship between repetitive head impacts, microglial activation, and neurodegeneration. Alzheimers Dement. 2018;14(9):1159-70.

37. Stern RA, Tripodis Y, Baugh CM, Fritts NG, Martin BM, Chaisson C, et al. Preliminary study of plasma exosomal tau as a potential biomarker for chronic traumatic encephalopathy. J Alzheimers Dis. 2016;51(4):1099-109

38. Shahim P, Tegner Y, Wilson DH, Randall J, Skillback T, Pazooki D, et al. Blood biomarkers for brain injury in concussed professional ice hockey players. JAMA Neurol. 2014;71(6):684-92.

39. Washnik NJ, Anjum J, Lundgren K, Phillips S. A review of the role of auditory evoked potentials in mild traumatic brain injury assessment. Trends Hear. 2019;23:2331216519840094.

40. Muraoka S, Jedrychowski MP, Tatebe H, DeLeo AM, Ikezu S, Tokuda T, et al. Proteomic profiling of extracellular vesicles isolated from cerebrospinal fluid of former national football league players at risk for chronic traumatic encephalopathy. Front Neurosci. 2019;13:1059.

41. Nedergaard M. Neuroscience. Garbage truck of the brain. Science. 2013:340(6140):1529-30.

42. Janelidze S, Stomrud E, Smith R, Palmqvist S, Mattsson N, Airey DC, et al. Cerebrospinal fluid p-tau217 performs better than p-tau181 as a biomarker of Alzheimer's disease. Nat Commun. 2020;11(1):1683.

43. Simren J, Leuzy A, Karikari TK, Hye A, Benedet AL, Lantero-Rodriguez J, et al. The diagnostic and prognostic capabilities of plasma biomarkers in Alzheimer's disease. Alzheimers Dement. 2021;17(7):1145-56.

44. Ashton NJ, Pascoal TA, Karikari TK, Benedet AL, Lantero-Rodriguez J, Brinkmalm G, et al. Plasma p-tau231: a new biomarker for incipient Alzheimer's disease pathology. Acta Neuropathol. 2021;141(5):709-24.

\section{Publisher's Note}

Springer Nature remains neutral with regard to jurisdictional claims in published maps and institutional affiliations. 